\title{
A Nonlinear Diffusion Filter Model to Enhance Infrared Multi-Wave- Band Finger Vein Images
}

\author{
Chen Liukui ${ }^{1}$, Li Zuojin ${ }^{* 1}$, Wu Ying ${ }^{1}$, Feng Lixiao ${ }^{1}$ \\ ${ }^{1}$ Chongqing University of Science and Technology \\ Huxi Street 200\#, Chongqing, 401331, P.R. China \\ E-mail of Corresponding author Li Zuojin*: cqustlzj@126.com
}

Keywords: infrared multi-wave-band finger vein images, joint structure tensor, nonlinear diffusion.

\begin{abstract}
This paper presents a structure tensor to nonlinear diffusion filtering for the infrared multi-band finger vein images to solve the lower contrast and uneven gray distribution in the infrared single band finger vein image. This joint structure tensor makes the nonlinear diffusion along the vein tendency, the structure preserving of vein edge and removing the speckle noise. In the examination, the proposed method shows the enhanced result.
\end{abstract}

\section{Introduction}

Finger vein recognition is based on the data of its infrared image[1][2][3]. The finger vein image of single-band infrared angiography has poor quality, which is presented as fuzzy vein edge, low contrast and gray non-uniform distribution. In this paper an infrared three band lighting and imaging is employed to obtain more comprehensive vein information than single band. This paper presents capturing the infrared multi-wave-band finger vein images(IMWBFVI), in which the veins has this strong spatial correlation and complementary, and more abundant vein information than single-band imaging. The vein has strong flow-like feature, which can be enhanced by the non-linear diffusion filter of Partial Differential Equation(PDE)..

Vein pattern is featured with its obvious line-shape, which could be satisfactorily detected by the structure tensor of PDE. The joint structure tensor of IMWBHVI is used to construct diffusion tensor for each image. This uniform diffusion tensor makes the nonlinear diffusion: the filter diffuses along the vein tendency in each band finger vein image. The structure preserving of vein edge and bifurcation is realized in the filtering noise in order to avoid the blurring of edges and other localization problems presented by linear diffusion models.

\section{Capture Infrared Multi-Band Finger Vein Images}

Nowadays, the capturing peak band of capture is $850 \mathrm{~nm}$ and the width is about $50 \mathrm{~nm}$ in the infrared single band imaging. In this paper, three infrared band LEDs are employed in the lighting source to capture the finger vein images. The parameters of three bands are shown in the figure 1 . Refer to paper [4], the examples of infrared multi-wave-band finger vein images are shown in the figure 2. These three-band images have various gray distribution and many speckle noises. The vein edge is blurry, which is difficult to enhancement.

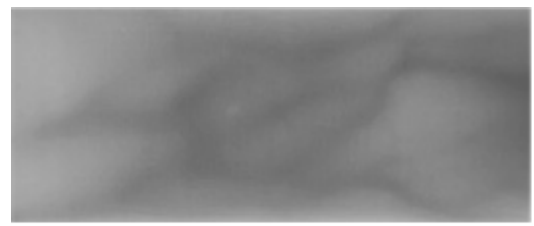

(a) finger vein image of $760 \mathrm{~nm}$ band

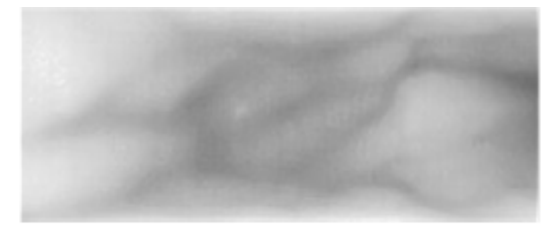

(b) finger vein image of $850 \mathrm{~nm}$ band

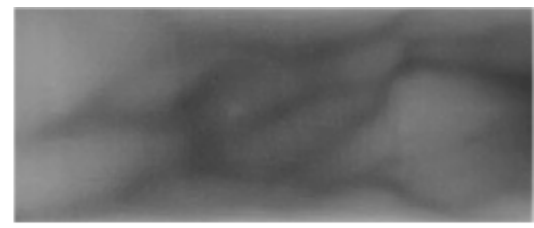

(c) finger vein image of $940 \mathrm{~nm}$ band

Figure 1. Example of infrared three band finger vein images 


\section{Capture Infrared Multi-Band Finger Vein Images}

The classic coherence-enhance model to incorporate local information from an origin image was proposed by Joachim Weickert[5], which is a nonlinear diffusion called 'anisotropic'. This paper presents the nonlinear diffusion filtering principle of infrared multi-band finger vein images: one band finger vein image is denoted as $I^{(i)}(x, y) \quad i=1,2,3$, One calculates a processed version $u^{(i)}(x, y, t)$ of $I^{(i)}(x, y)$ with a scale parameter $t \geq 0$ as the solution of a diffusion equation with the origin image $I^{(i)}(x, y)$ as initial condition and reflecting boundary conditions:

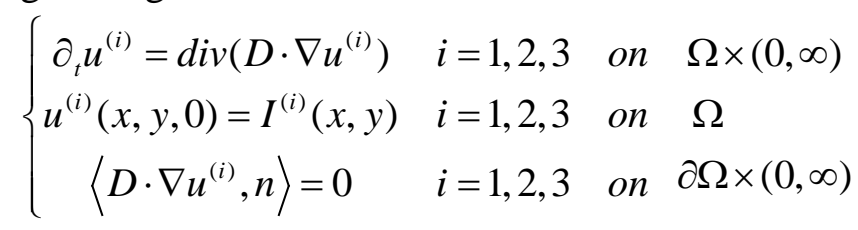

By the initial condition $t=0, u^{(i)}(x, y, 0)=I^{(i)}(x, y)$, n denotes the outer normal and $\langle\square, \square\rangle$ the usual inner product. $\mathrm{D}$ is defined as the diffusion tensor, which is created and based on the structure tensor of $u^{(i)}(x, y, t) \quad i=1,2,3$. This structure tensor is a powerful tool for analyzing coherence structures. The joint structure tensor ${ }^{J_{\rho}}$ in this paper is defined as:

$$
\left\{\begin{array}{cc}
J_{\rho}=K_{\rho} *\left(\sum_{i=1}^{3} \nabla u_{\sigma}^{(i)} \otimes \nabla u_{\sigma}^{(i)}\right) & i=1,2,3 \\
u_{\sigma}^{(i)}=K_{\sigma} * u^{(i)} & i=1,2,3
\end{array}\right.
$$

Every component of the resulted matrix of the tensor product is convolved with a Gaussian kernel $\left(K_{\rho}\right)$. The eigenvectors of the joint structure tensor $J_{\rho}$ represents the average orientation of the gradient vectors for the IMBFVI and the structure orientation at the scale $\rho$. The joint diffusion tensor $D=\left(\begin{array}{ll}d_{11} & d_{12} \\ d_{21} & d_{22}\end{array}\right)$ in (1) has the same eigenvectors as $J_{\rho}=\left(\begin{array}{ll}j_{11} & j_{12} \\ j_{21} & j_{22}\end{array}\right)$, but its eigenvalues $\mu_{1,2}$ are chosen according to a coherence measure. The trace and det of $J_{\rho}$ can be calculated as

$$
\left\{\begin{array}{l}
\operatorname{trace}\left(J_{\rho}\right)=j_{11}+j_{12} \\
\operatorname{det}\left(J_{\rho}\right)=j_{11} j_{12}-j_{12}{ }^{2}
\end{array}\right.
$$

Its eigenvalues can be calculated as;

$$
\mu_{1,2}=0.5 *\left(\operatorname{trace}\left(J_{\rho}\right) \pm \sqrt{\operatorname{trace}\left(J_{\rho}\right)^{2}-4 \operatorname{det}\left(J_{\rho}\right)}\right)
$$

The corresponding orthonormal set of eigenvectors $\left\{w_{1}, w_{2}\right\}$ is given by

$$
w_{1}=\left[\begin{array}{c}
\cos \phi \\
\sin \phi
\end{array}\right] \quad w_{2}=\left[\begin{array}{c}
\sin \phi \\
\cos \phi
\end{array}\right]
$$

In (5), the $\phi$ satisfies:

$$
\tan (2 \phi)=\frac{2 j_{12}}{j_{11}-j_{22}}
$$

The diffusion/smoothing process acts mainly along the structure direction and becomes stronger if the coherence increases in any band finger vein image. The coherence is denoted as $\kappa$. These can be achieved in the following way:

$$
\kappa=\left(j_{11}-j_{22}\right)^{2}+4 j_{12}^{2}
$$

The value of $\kappa$ becomes large for anisotropic structures. It measures the coherence within the scale of $\rho$, and its range is from 0 to $\infty$.

The diffusion matrix $D$ which has same eigenvectors as $J_{\rho}$ has the eigenvalues of follows: 


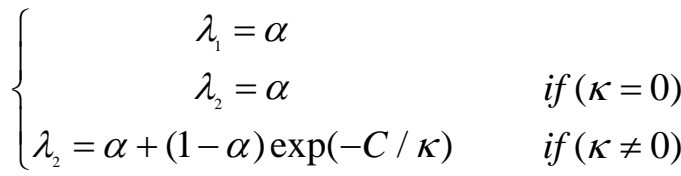

The range of parameter $\alpha$ is $(0,1)$, which keeps the diffusion tensor uniformly positive definite[6].

\section{Performance evaluation}

In this examination, The parameters of the proposed nonlinear diffusion filter are set as follows: $\alpha=0.01, \sigma=1, \rho=5, C=1.2564, \Delta t=0.3$. The total number of diffusion iterations is 300 , and the result images are shown in the figure 3 . The vessels are enhanced and the speckle noises are smoothed.

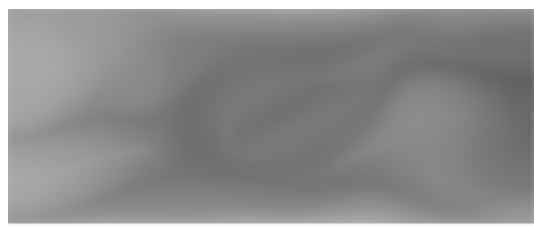

(a) 300 diffusion iteration result to $760 \mathrm{~nm}$

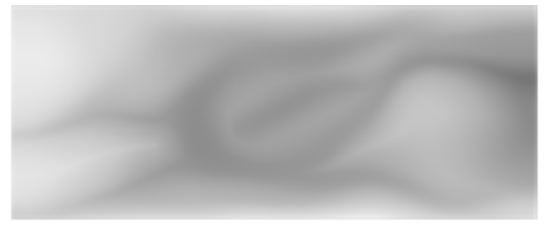

(b) 300 diffusion iteration result to $850 \mathrm{~nm}$

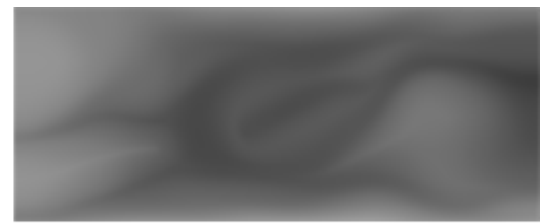

(c) 300 diffusion iteration result to $940 \mathrm{~nm}$

Figure 3 the 300 iteration diffusion result of the multi-wave-band infrared finger vein images

To compare the enhancement result between the proposed method and other methods[7], in which one is the linear Gaussian filter and another is non-linear median filter.

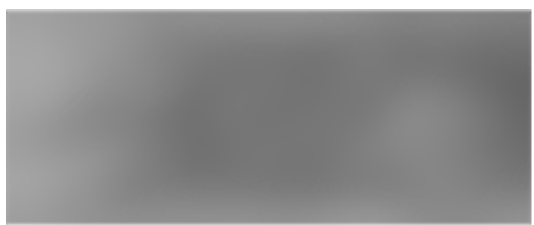

(a) 300 iteration filter of Gaussian to $760 \mathrm{~nm}$

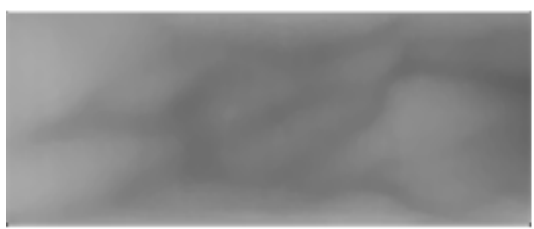

(d) 300 iteration filter of Gaussian to $760 \mathrm{~nm}$

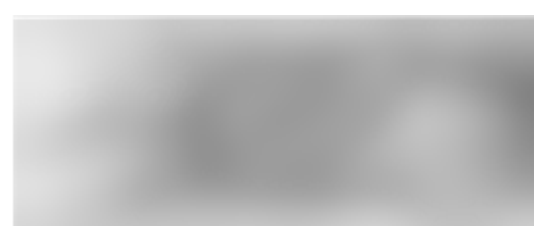

(b) 300 iteration filter of Gaussian to $850 \mathrm{~nm}$

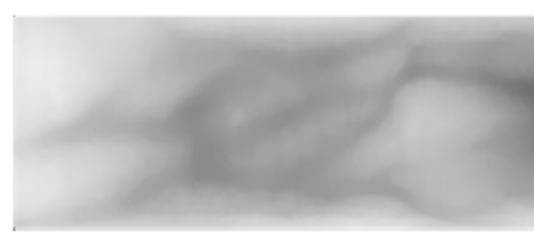

(e) 300 iteration filter of Gaussian to $850 \mathrm{~nm}$

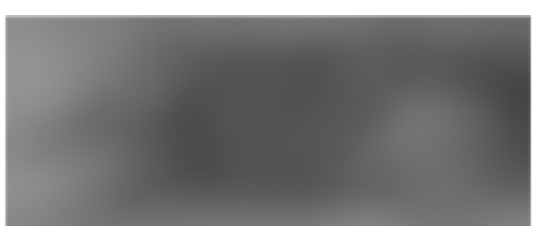

(c) 300 iteration filter of Gaussian to $940 \mathrm{~nm}$

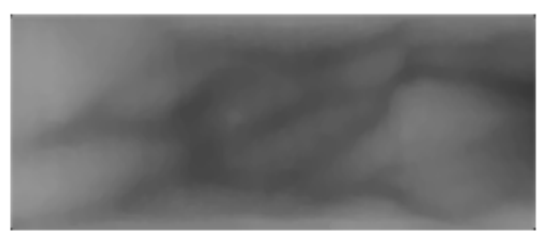

(f) 300 iteration filter of Gaussian to $940 \mathrm{~nm}$

Figure 4 the 300 iteration filter result of Gaussian and median to the multi-wave-band finger vein images

The proposed nonlinear filter is better than the linear Gaussian filter in the iteration operation. Here the average gradient of gray is calculated to present the vein edge information. The average gradient value of the proposed method, Gaussian and median filter is plot in the figure 6, in which the average gradient of the proposed nonlinear filter has better edge enhancement than median filter before the 150 iteration. The average gradient fallen rate of Gaussian filter is the fastest while iterating filter. 


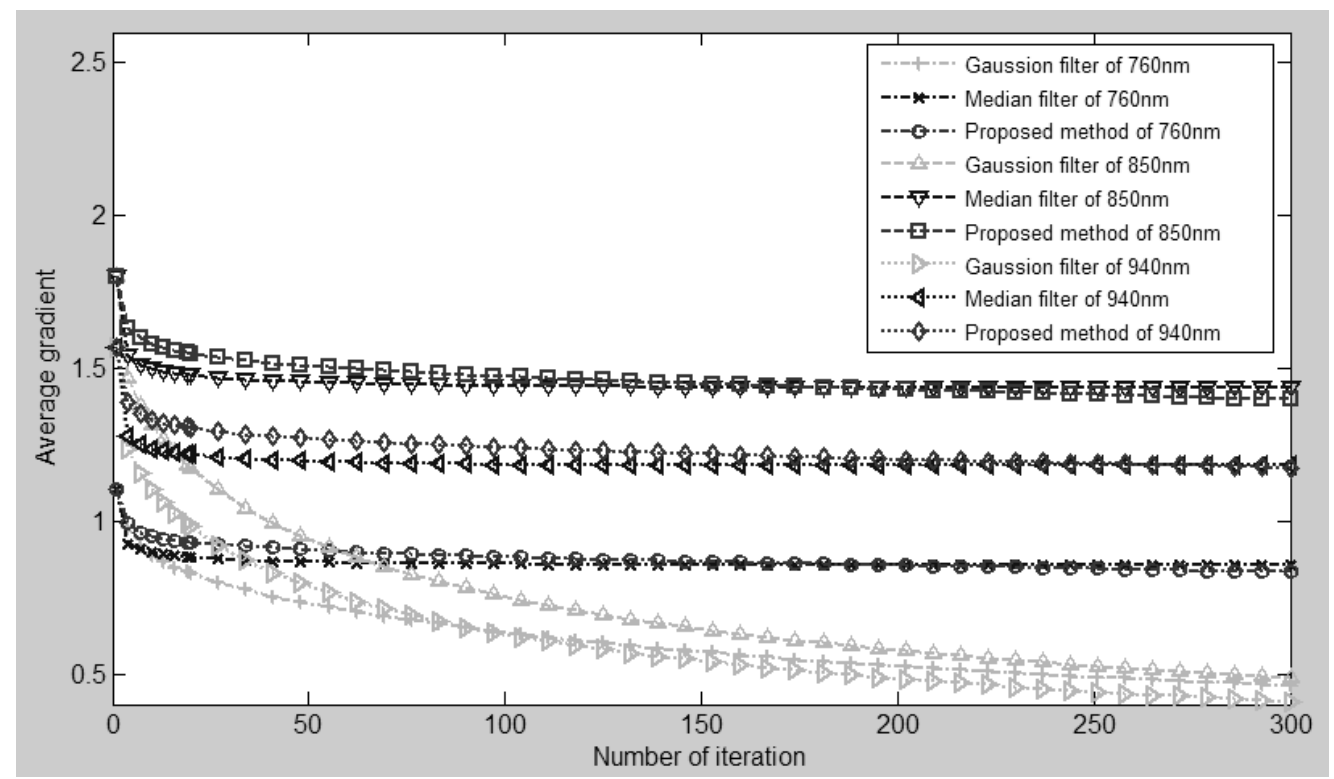

Figure 6 the average gradient of the Gaussian, median and proposed nonlinear filter in the iteration operation to the $760 \mathrm{~nm}, 850 \mathrm{~nm}$ and $940 \mathrm{~nm}$ infrared band finger vein images

\section{Conclusion}

It can be seen from figures and examinations, the proposed joint scattering matrix to nonlinear diffusion filtering for the infrared multi-band finger vein images is a convenient method to enhance finger vein images. The further work is to search the optimization parameter of the diffusion and the multi-band finger vein images' fusion.

\section{Acknowledgment}

The authors would like to express their gratitude and thanks to Natural Science Foundation Project of CQ CSTC(cstcjjA40041, cstc2014jcyjA40006), Science Technolgy Reseach Project of CQJW(KJ131422, KJ131423) and Campus Research Foundation of Chongqing University of Science and Technology (CK2011B09, CK2011B05) for their financial support to this research work.

\section{References}

[1] Park, Young Ho; Park, Kang Ryoung, Image Quality Enhancement Using the Direction and Thickness of Vein Lines for Finger-Vein Recognition, INTERNATIONAL JOURNAL OF ADVANCED ROBOTIC SYSTEMS, OCT 29 ${ }^{\text {th }} 2012$, Volumn 9

[2] Yang, Jinfeng; Shi, Yihua, Finger-vein ROI localization and vein ridge enhancement, PATTERN RECOGNITION LETTERS, SEP 2012, 33(12), pp: 1569-1579

[3] N. Miura, A. Nagasaka, T. Miyatake. Extraction of Finger-Vein Patterns Using Maximum Curvature Points in Image Profiles, IEICE TRANS. INF. \& SYST., VOL.E90-D, NO.8 ,AUGUST 2007, pp:1185-1194.

[4] Chen, Liu Kui; Li, Zuo Jin; Wu, Ying; Xiang, Yi; Dynamic Range Extend on Finger Vein Image Based on Infrared Multi-Light-Intensity Vascular Imaging, Applied Mechanics and Materials, v 427-429, p 1832-1835, 2013

[5] Joachim Weickert. Coherence-Enhancing Diffusion Filtering[J]. International Journal of Computer Vision . 1999 (2-3) pp:111-127.

[6] Cheng Jiangang, Tian Jie, He Yuliang, Yang Xin, Fingerprint Enhancement Algorithm Based on Nonlinear Diffusion Filter, Acta Automatica Sinica, 2004, 30(6),pp:854-862.

[7] Z. Wang, A. C. Bovik, H. R. Sheikh, and E. P. Simoncelli. Image quality assessment: From error visibility to structural similarity. IEEE Transaction on Image Processing, 2004, 13(4):600-612 